\title{
IL-4 and IL-12 Polymorphisms are Associated with Response to Suplatast Tosilate, a Th2 Cytokine Inhibitor, in Patients with Atopic Dermatitis
}

\author{
Hideki Nagase ${ }^{1}$, Yoshinori Nakachi ${ }^{1}$, Keiji Ishida ${ }^{1}$, Mamoru Kiniwa ${ }^{*}$, , Satoshi Takeuchi ${ }^{2}$, Ichiro \\ Katayama $^{3}$, Yoshinari Matsumoto ${ }^{4}$, Fukumi Furukawa ${ }^{5}$, Shin Morizane ${ }^{6}$, Sakae Kaneko ${ }^{7}$, Yoshiki \\ Tokura $^{8}$, Motoi Takenaka ${ }^{9}$, Yutaka Hatano ${ }^{10}$ and Yoshiki Miyachi ${ }^{11}$ \\ ${ }^{1}$ Tokushima Research Center, Taiho Pharmaceutical Co., Ltd., Tokushima, Japan \\ ${ }^{2}$ Department of Dermatology, Graduate School of Medical Sciences, Kyushu University, Fukuoka, Japan \\ ${ }^{3}$ Department of Dermatology, Osaka University Graduate School of Medicine, Osaka, Japan \\ ${ }^{4}$ Department of Dermatology, Aichi Medical University School of Medicine, Aichi, Japan \\ ${ }^{5}$ Department of Dermatology, Wakayama Medical University, Wakayama, Japan \\ ${ }^{6}$ Department of Dermatology, Okayama University Graduate School of Medicine, Dentistry and Pharmaceutical \\ Sciences, Okayama, Japan \\ ${ }^{7}$ Department of Dermatology, Shimane University Faculty of Medicine, Shimane, Japan \\ ${ }^{8}$ Department of Dermatology, University of Occupational and Environmental Health, Kitakyushu, Japan (Present \\ affiliation: Department of Dermatology, Hamamatsu University School of Medicine, Hamamatsu, Japan) \\ ${ }^{9}$ Department of Dermatology and Allergology, Nagasaki University Hospital, Nagasaki, Japan \\ ${ }^{10}$ Department of Dermatology, Faculty of Medicine, Oita University, Oita, Japan \\ ${ }^{11}$ Department of Dermatology, Kyoto University Graduate School of Medicine, Kyoto, Japan
}

\begin{abstract}
Th2-related immune and inflammatory responses have been implicated in the pathogenesis of atopic dermatitis (AD), but few clinical lines of evidence have been reported regarding how and whether Th2-related responses are associated with other risk factors in the treatment of $\mathrm{AD}$ patients. In this study, the associations between the polymorphisms of genes related to the pathophysiology of AD and the efficacy of suplatast tosilate, an oral immunemodulator known to downregulate Th2-related allergic responses, were analyzed in adult patients with chronic AD. Patients were recruited from our previous study, where suplatast tosilate was evaluated for its efficacy when used in combination with topical steroids. The genotypes of 35 single nucleotide polymorphisms (SNPs) of 27 genes related to $\mathrm{AD}$ pathogenesis were then determined in 17 responders and 18 non-responders, as defined by the improvement rate in their AD skin scores. While no significant difference in the patient background was observed between responders and non-responders, significant associations were noted between the response to treatment with suplatast tosilate and three SNPs of IL-4 (-590C/T: $P=0.04,-33 \mathrm{C} / \mathrm{T}: P=0.04)$ and IL-12B $(1188 \mathrm{~A} / \mathrm{C}: P=0.03)$, but not for the other SNPs. Of note, ethnic differences in the genotype frequencies of IL-4 -590C/T and IL-12B 1188A/C SNPs were found. In conclusion, the present results raise the possibility that $\mathrm{AD}$ patients who tend to produce more IL-4 and IL-12 may be susceptible to suplatast tosilate treatment and that ethnic variations should be considered to further understand the role of Th2-related responses.
\end{abstract}

Keywords: Atopic dermatitis, single nucleotide polymorphism, IL-4, IL-12B, Th1, Th2, suplatast tosilate, ethnic difference.

\section{INTRODUCTION}

$\mathrm{AD}$ is one of the most common chronic inflammatory diseases of the skin. While its causes and mechanisms have not yet been fully elucidated, its pathogenesis has been

*Address correspondence to this author at the Tokushima Research Center, Taiho Pharmaceutical Co., Ltd., 224-2, Ebisuno Hiraishi, Kawauchi-cho, Tokushima, 771-0194, Japan; Tel: +81-88-665-6014; Fax: +81-88-6656554; E-mail: makiniwa@taiho.co.jp characterized by altered skin barrier function and immune dysregulation. Recent genome-wide association studies or polymorphism analyses of candidate genes have further extended the view that patients with $\mathrm{AD}$ have genetically determined risk factors that affect the skin barrier and immune responses [1-6]. It should be noted, however, that few clinical lines of evidence have been reported regarding how these factors interact with each other and, more importantly, whether the down- or up-regulation of any gene or factor may affect the treatment of AD patients. 
In the present study, we focused on suplatast tosilate, which has been approved in Japan as an oral immunemodulator for $\mathrm{AD}$, allergic rhinitis, and asthma [7-10]. This agent has been used as an adjunct to or in combination with topical anti-inflammatory and immunosuppressive agents and has been shown to reduce the requirements for these agents $[11,12]$. Suplatast tosilate has been referred to as a Th2 cytokine inhibitor because 1) it was discovered based on its capacity to suppress murine $\operatorname{IgE}$ formation without affecting cellular immune responses [13], 2) it suppressed IL-4 and IL-5 production by Th2 cells [14], 3) it suppressed allergic inflammation via indirect actions on Th2 cells as well as direct actions on eosinophils [15-19], and 4) it affected certain biomarkers such as $\mathrm{Th} 1 / \mathrm{Th} 2$ ratio during the treatment of allergic asthmatic patients [20]. Although its mechanism of action remains to be identified at a molecular level, it is reasonable to assume that its efficacy in allergic patients may be ascribed to the modulation of the Th2related immune and inflammatory responses.

We previously performed a clinical study investigating the efficacy of suplatast tosilate in adult chronic AD patients who also received topical steroids but had responded poorly to adjunct therapy such as antihistamines [21,22]. This study revealed that suplatast tosilate markedly improved the AD skin symptom scores in a certain group of patients, but not in all patients, enabling us to recruit suplatast tosilate responders and non-responders and to analyze the polymorphism of genes related to the pathophysiology of AD retrospectively. The results implicated the efficacy of suplatast tosilate in the modulation of both Th1 and Th2 responses in AD therapy and suggested ethnic variations in the pathogenesis of $\mathrm{AD}$.

\section{MATERIALS AND METHODS}

\section{Study Subjects}

One hundred and sixty-three patients with atopic dermatitis whose conditions had been poorly controlled by other anti-allergic drugs at 11 hospitals between 2004 and 2006 were enrolled in our previous study [21, 22]. The Th2 cytokine inhibitor suplatast tosilate (300 $\mathrm{mg} /$ day) was administered to these patients. After 4 weeks or more of treatment, the skin symptom score was determined to evaluate the usefulness of suplatast tosilate. The effectiveness of suplatast tosilate was evaluated by comparing the improvement rate relative to the baseline skin symptom score $[21,22]$. To classify the patients into a highly effective group and a poorly effective group, we divided all the cases into three groups based on the improvement rate of the skin symptom scores. The one-third of cases with the highest improvement rates were defined as "responders", and the one-third of cases with the lowest improvement rates were defined as "non-responders". As a result, 54 "responder" patients had an improvement rate of more than $58 \%$, and 53 "non-responder" patients had an improvement rate of less than $25 \%$. From these patients, a total of 35 patients (17 responders and 18 non-responders) provided informed consent to undergo SNP genotyping using DNA from a blood sample. The improvement rate for the 17 highresponders with an improvement rate of more than 58\% for their skin symptom scores was $68.8 \pm 12.2 \%$. On the other hand, that of the 18 non-responders with an improvement rate of less than $25 \%$ was $1.4 \pm 22.8 \%$. This study was approved by the ethics committees of Kyoto University Graduate School of Medicine, Kyushu University, Osaka University Graduate School of Medicine, Aichi Medical University School of Medicine, Wakayama Medical University, Okayama University Graduate School of Medicine, Shimane University, University of Occupational and Environmental Health, Nagasaki University, Oita University and Taiho Pharmaceutical Co., Ltd.

\section{SNP Genotyping Analysis}

In each patient, $8.5 \mathrm{~mL}$ of blood was collected into a PAXgene Blood DNA tube (Qiagen Inc. CA, USA). DNA from the blood sample was purified using a PAXgene Blood DNA kit (Qiagen Inc.). The genotypes of 35 single nucleotide polymorphisms (SNPs) of 27 allergy-related genes were determined using a TaqMan real-time polymerase chain reaction (PCR) (TaqMan SNP Genotyping Assays; Life Technologies, CA, USA) or direct sequencing methods. The following 27 genes were investigated in this study: Th2 cytokines or cytokines promoting Th2 cells (IL-4, IL-5, IL-13, and TSLP), Th1 cytokine (IFN- $\gamma$ ), cytokines promoting Th1 cells (IL-12B and IL-18), proinflammatory cytokine (IL-17), chemokines (CCL5, CCL11, and CCL17), some of their receptors (IL-4R, IL-5R, IL-12R, IL-22R, IL$23 \mathrm{R}$, and IFN- $\gamma \mathrm{R})$, other genes related to the symptoms of $\mathrm{AD}$ or atopic disease (IL-31R, Chymase, NGF, NGFR, FceR1 $\alpha$, FceR1 $\beta$, HRH1, HRH4, and LTC4S), and filaggrin (FLG). Thirty-one of the 35 SNPs were genotyped using the Applied Biosystems 7900HT Real-Time PCR System. The assay IDs of the 31 SNPs are shown in Table 1. The remaining four SNPs without a commercial TaqMan SNP genotyping assay were genotyped by direct sequencing at Takara Bio Inc. (Mie, Japan).

\section{Statistical Analysis}

The associations between the clinical parameters and the response to treatment with suplatast tosilate were evaluated using a likelihood ratio chi-square test for categorical data or the Student t-test for continuous data. The effect of suplatast tosilate on the clinical parameters was evaluated using a paired $t$-test. Hardy-Weinberg equilibrium of the genotyping results was evaluated using a Pearson's chi-square test. A likelihood ratio chi-square test was used to analyze the associations between the genotype of each SNP and the response to treatment with suplatast tosilate. All the analyses were performed using the statistical software JMP 7.0.1 and the SAS statistical package, version 9.1.3 (SAS Institute Inc., Cary, NC, USA). Differences were considered significant when $P<0.05$. Because this was the study with a small sample size, a correction for multiple comparisons was not made.

\section{RESULTS}

\section{Patient Characteristics and Effect of Suplatast Tosilate on Th2-Related Parameters}

No significant differences between the responders and the non-responders were observed with regard to sex, age, and the pre-treatment values for the skin symptom scores, IgE levels or eosinophil counts (Table 2). Note that suplatast tosilate treatment did not affect the serum IgE level or the 
Table 1. SNP list and Genotyping Methods

\begin{tabular}{|c|c|c|c|c|}
\hline Gene Symbol & SNP name & rs ID & Methods & Assay ID \\
\hline \multirow[t]{2}{*}{ IL4 } & $-590 \mathrm{C} / \mathrm{T}$ & rs2243250 & RT-PCR* & C_16176216_10 \\
\hline & $-33 \mathrm{C} / \mathrm{T}$ & rs2070874 & RT-PCR & C_16176215_10 \\
\hline \multirow[t]{2}{*}{ IL4R } & Gln551Arg & rs 1801275 & RT-PCR & C__2351160_20 \\
\hline & Ile50Val & rs1805010 & RT-PCR & C__2769554_10 \\
\hline IL5 & $-703 \mathrm{C} / \mathrm{T}$ & rs2069812 & RT-PCR & C_16274150_10 \\
\hline IL5RA & $-80 \mathrm{G} / \mathrm{A}$ & rs2290608 & RT-PCR & C_15885096_10 \\
\hline IL12B & $1188 \mathrm{~A} / \mathrm{C}$ & rs3212227 & RT-PCR & C__2084293_10 \\
\hline IL12RB1 & $-2 \mathrm{C} / \mathrm{T}$ & rs 436857 & RT-PCR & C__ 795468_1_ \\
\hline \multirow[t]{2}{*}{ IL13 } & 2044G/A & rs 20541 & RT-PCR & C__2259921_20 \\
\hline & $-1112 \mathrm{C} / \mathrm{T}$ & rs 1800925 & RT-PCR & C__8932056_10 \\
\hline IL17F & $7488 \mathrm{~T} / \mathrm{C}$ & rs763780 & RT-PCR & C__2234166_10 \\
\hline IL18 & $113 \mathrm{~T} / \mathrm{G}$ & rs 360718 & RT-PCR & C__2898461_10 \\
\hline IL22RA1 & Arg518Gly & rs3795299 & RT-PCR & C__ $440166 \_10$ \\
\hline IL23R & Gln3His & rs1884444 & RT-PCR & C_11728603_10 \\
\hline IL31RA & Ser497Asn & rs161704 & RT-PCR & C__2839337_10 \\
\hline IFNG & $874 \mathrm{~T} / \mathrm{A}$ & rs2430561 & $\mathrm{DS} \dagger$ & - \\
\hline IFNGR2 & Arg64Gln & rs9808753 & RT-PCR & C__2443413_1_ \\
\hline \multirow[t]{2}{*}{ CCL5 } & $-28 \mathrm{C} / \mathrm{G}$ & rs2280788 & RT-PCR & C_15874396_20 \\
\hline & $-403 \mathrm{G} / \mathrm{A}$ & rs2107538 & RT-PCR & C_15874407_10 \\
\hline CCL11 & $-384 \mathrm{~A} / \mathrm{G}$ & rs17809012 & RT-PCR & C__ 2590323_10 \\
\hline CCL17 & $-431 \mathrm{C} / \mathrm{T}$ & rs 223828 & RT-PCR & C__2392392_10 \\
\hline TSLP & $-847 \mathrm{C} / \mathrm{T}$ & rs3806933 & RT-PCR & C__3166722_10 \\
\hline NGFB & Ala35Val & rs6330 & RT-PCR & C__2525309_10 \\
\hline NGFR & Ser205Leu & rs2072446 & RT-PCR & C_15870920_10 \\
\hline FCER1A & $-66 \mathrm{~T} / \mathrm{C}$ & rs2251746 & RT-PCR & C__1840470_20 \\
\hline \multirow[t]{2}{*}{ MS4A2 } & $-109 \mathrm{C} / \mathrm{T}$ & rs1441586 & RT-PCR & C__1842226_10 \\
\hline & Glu237Gly & rs569108 & RT-PCR & C__ $900116 \_10$ \\
\hline CMA1 & $-1897 \mathrm{G} / \mathrm{A}$ & rs 1800875 & RT-PCR & C__ 2796262_10 \\
\hline HRH1 & $-17 \mathrm{C} / \mathrm{T}$ & rs901865 & RT-PCR & C_25471612_10 \\
\hline HRH4 & Ala138Val & rs11665084 & RT-PCR & C__3161321_20 \\
\hline LTC4S & $-444 \mathrm{~A} / \mathrm{C}$ & rs 730012 & RT-PCR & C__644967_10 \\
\hline \multirow[t]{4}{*}{ FLG } & Tyr2194His & rs 2184953 & DS & - \\
\hline & Tyr3105Asp & rs2065958 & DS & - \\
\hline & $13347 \mathrm{G} / \mathrm{A}$ & rs12730241 & RT-PCR & C_31910001_10 \\
\hline & $13586 \mathrm{C} / \mathrm{T}$ & rs11204976 & DS & - \\
\hline
\end{tabular}

*RT-PCR: reverse transcription polymerase chain reaction,

$\dagger$ DS: direct sequencing.

eosinophil count in either the responders or the nonresponders (Table $\mathbf{3}$ ).

\section{Genotype and Allele Frequencies}

The genotyping results and the allele frequencies of the 35 SNPs are shown in Table 4. Among 34 SNPs (excluding the Filaggrin Tyr3105Asp SNP), the genotype frequencies were the values expected under Hardy-Weinberg equilibrium, and no significant difference was observed between the minor allele frequencies reported in this study and those shown in the SNPper (http://snpper.chip.org/bio/snpperenter) or NCBI databases (http://www.ncbi.nlm.nih.gov/ $\mathrm{snp} /$ ) (data not shown), indicating that the genotyping 
Table 2. Clinical Characteristics of the Patients

\begin{tabular}{|l|c|c|c|}
\hline \multicolumn{1}{|c|}{ Parameters } & Responders (n=17) & Non-Responders (n=18) & P-Value* \\
\hline \hline $\begin{array}{l}\text { Sex } \\
\text { Male/Female }\end{array}$ & $11 / 6$ & $10 / 8$ & 0.58 \\
\hline $\begin{array}{l}\text { Age } \\
\text { (Range) }\end{array}$ & $37.1 \pm 17.5$ & $32.3 \pm 12.6$ & $(13-62)$ \\
\hline $\begin{array}{l}\text { Skin symptom score (Pre) } \\
\text { (Range) }\end{array}$ & $(18-79)$ & $10.4 \pm 4.0$ & 0.36 \\
\hline $\begin{array}{l}\text { IgE level (Pre) } \\
(\text { IU/mL) }\end{array}$ & $(4-6 \pm 2.8$ & $(3-17)$ & 0.12 \\
\hline $\begin{array}{l}\text { Eosinophil count (Pre) } \\
\left(\text { cells/mm }{ }^{3}\right)\end{array}$ & $8645 \pm 8429$ & $16356 \pm 21988$ & 0.20 \\
\hline $\begin{array}{l}\text { Eosinophil count (Pre) } \\
(\%)\end{array}$ & $615 \pm 387$ & $581 \pm 329$ & 0.80 \\
\hline
\end{tabular}

*The $P$-values for categorical data and for continuous data were obtained using the likelihood ratio chi-square test and Student t-test, respectively.

Table 3. Effect of Suplatast Tosilate on Skin Symptom Score, Serum IgE Level, and Eosinophil Count in Atopic Dermatitis Patients

\begin{tabular}{|c|c|c|c|c|c|}
\hline Parameters & & $\mathbf{N}$ & Pre-Treatment & Post-Treatment & $P$-Value \\
\hline $\begin{array}{c}\text { Skin symptom } \\
\text { score }\end{array}$ & Responders & 17 & $8.6 \pm 2.8$ & $2.8 \pm 1.5$ & $<0.001$ \\
\hline \multirow{2}{*}{$\begin{array}{l}\text { IgE level } \\
(\mathrm{IU} / \mathrm{mL})\end{array}$} & Responders & 16 & $8645 \pm 8429$ & $7935 \pm 8605$ & 0.29 \\
\hline & Non-responders & 14 & $16356 \pm 21988$ & $15047 \pm 19624$ & 0.09 \\
\hline $\begin{array}{l}\text { Eosinophil } \\
\left(\text { cells } / \mathrm{mm}^{3}\right)\end{array}$ & Non-responders & 14 & $581 \pm 329$ & $722 \pm 527$ & 0.28 \\
\hline \multirow{2}{*}{$\begin{array}{c}\text { Eosinophil } \\
(\%)\end{array}$} & Responders & 15 & $10.3 \pm 5.1$ & $9.1 \pm 5.4$ & 0.18 \\
\hline & Non-responders & 13 & $8.6 \pm 3.6$ & $10.4 \pm 6.4$ & 0.29 \\
\hline
\end{tabular}

$* P$-values were obtained using a paired $t$-test.

analysis was performed accurately. Regarding the genotyping results for the Filaggrin Tyr3105Asp SNP, the genotype frequency deviated from the values expected from Hardy-Weinberg equilibrium, and the minor allele frequency was significantly higher than that shown in the SNPper database (data not shown). This analysis may not have been performed accurately because the primer design region for the Filaggrin Tyr3105Asp SNP was highly homologous with other genes.

\section{Associations between Genotype and Response to Treatment with Suplatast Tosilate}

Significant associations between the response to treatment with suplatast tosilate and three SNPs of IL-4 (590C/T: $P=0.04,-33 \mathrm{C} / \mathrm{T}: P=0.04)$ and $\mathrm{IL}-12 \mathrm{~B}(1188 \mathrm{~A} / \mathrm{C}$ : $P=0.03)$ were observed among the 35 SNPs genotyped in this study (Table 4). The genotyping results of two SNPs ($590 \mathrm{C} / \mathrm{T}$ and $-33 \mathrm{C} / \mathrm{T})$ within the IL-4 promoter region were perfectly identical among the 35 patients in this study. Therefore, the IL-4 -590C/T SNP, as a representative of the two SNPs of IL-4 that showed a significant association with the response, was used in further analyses. For the IL-4 -
590C/T SNP, patients with a C/C genotype had a significantly lower response rate to suplatast tosilate than those with a $\mathrm{T} / \mathrm{T}$ or $\mathrm{T} / \mathrm{C}$ genotype $(0.0 \%$ vs $53.1 \%, P=0.04)$ (Table 5). On the other hand, for the IL-12B 1188A/C SNP, patients with an A/A genotype had a significantly lower response rate to suplatast tosilate than those with a $\mathrm{C} / \mathrm{C}$ or C/A genotype $(12.5 \%$ vs $59.3 \%, P=0.02$, odds ratio=10.2) (Table 5). In a combination analysis of the two SNPs (IL-4 $590 \mathrm{C} / \mathrm{T}$ and IL-12B 1188A/C), patients with either IL-4 $590 \mathrm{C} / \mathrm{T} \mathrm{C} / \mathrm{C}$ or IL-12B $1188 \mathrm{~A} / \mathrm{C} \mathrm{A} / \mathrm{A}$ had a significantly lower response rate than those with other genotype combinations $(10.0 \%$ vs $64.0 \%, P=0.002$, odds ratio=16.0 $)$ (Table 5).

\section{Ethnic Differences in Genotype Frequency of IL-4 - 590C/T and IL-12B 1188A/C SNPs}

The genotype frequencies of IL-4 -590C/T T/T and IL$12 \mathrm{~B} 1188 \mathrm{~A} / \mathrm{C} \mathrm{C} / \mathrm{C}$, which were associated with a strong response to suplatast tosilate, were significantly higher among Asians than among Europeans based on the results of the HapMap project (Table 6, http://www.ncbi.nlm.nih.gov/ snp/). 
Table 4. Genotype Frequency and Association Between Genotype and Response to Treatment with Suplatast Tosilate

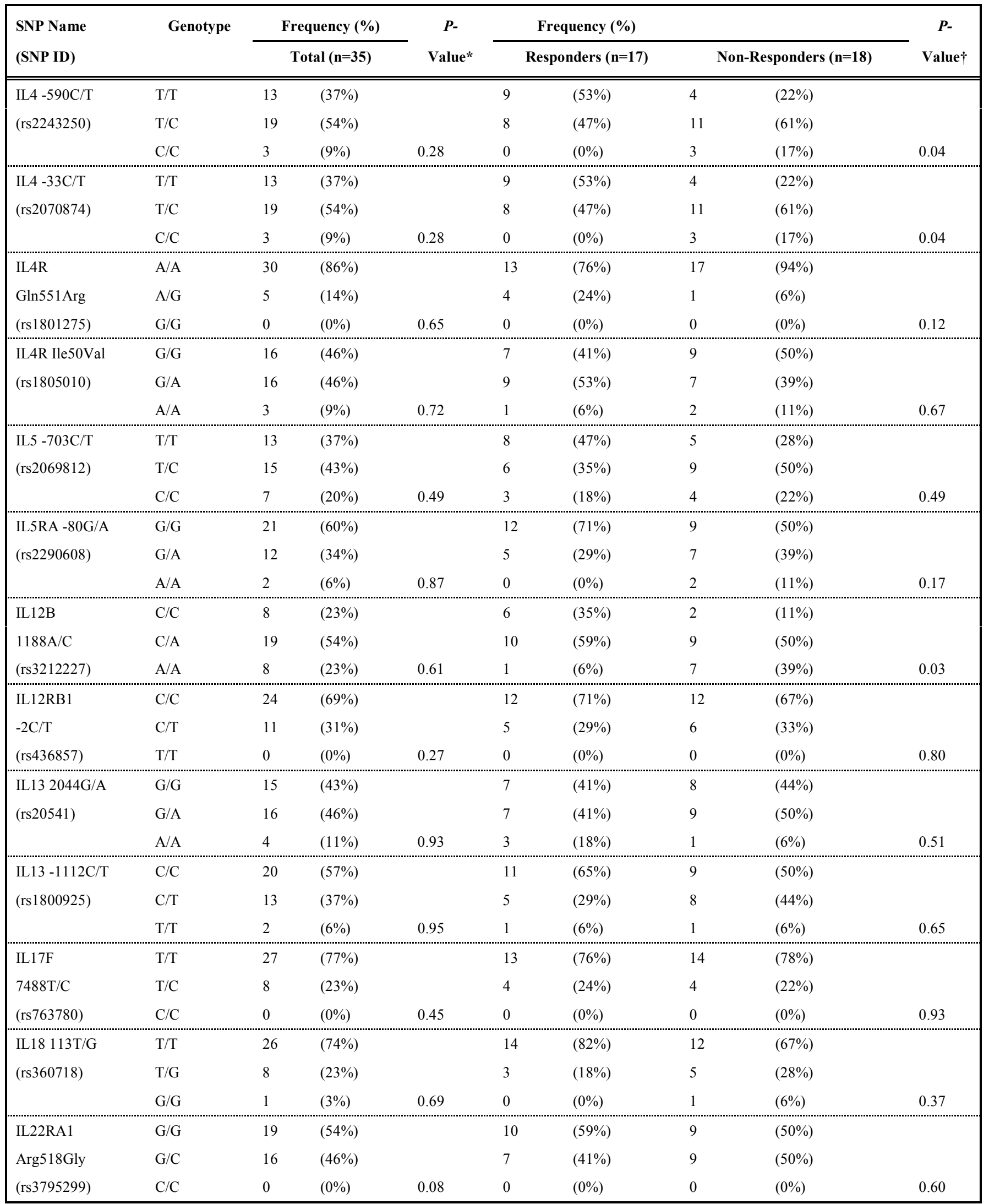


(Table 4) contd.....

\begin{tabular}{|c|c|c|c|c|c|c|c|c|c|}
\hline \multirow{3}{*}{$\begin{array}{l}\text { SNP Name } \\
\text { (SNP ID) } \\
\text { IL23R }\end{array}$} & \multirow{3}{*}{$\begin{array}{l}\text { Genotype } \\
\mathrm{T} / \mathrm{T}\end{array}$} & \multirow{2}{*}{\multicolumn{2}{|c|}{$\frac{\text { Frequency }(\%)}{\text { Total }(\mathrm{n}=\mathbf{3 5})}$}} & \multirow{3}{*}{$\begin{array}{c}P- \\
\text { Value* }\end{array}$} & \multicolumn{4}{|c|}{ Frequency $(\%)$} & \multirow{4}{*}{$\begin{array}{c}P- \\
\text { Value }\end{array}$} \\
\hline & & & & & \multicolumn{2}{|c|}{ Responders ( $\mathrm{n}=17)$} & \multicolumn{2}{|c|}{ Non-Responders $(\mathrm{n}=18)$} & \\
\hline & & 11 & $(31 \%)$ & & 4 & $(24 \%)$ & 7 & $(39 \%)$ & \\
\hline Gln3His & $\mathrm{T} / \mathrm{G}$ & 20 & $(57 \%)$ & & 10 & $(59 \%)$ & 10 & $(56 \%)$ & \\
\hline$(\mathrm{rs} 1884444)$ & $\mathrm{G} / \mathrm{G}$ & 4 & $(11 \%)$ & 0.26 & 3 & $(18 \%)$ & 1 & $(6 \%)$ & 0.40 \\
\hline IL31RA & $\mathrm{G} / \mathrm{G}$ & 9 & $(26 \%)$ & & 6 & $(35 \%)$ & 3 & $(17 \%)$ & \\
\hline Ser497Asn & $\mathrm{G} / \mathrm{A}$ & 17 & $(49 \%)$ & & 6 & $(35 \%)$ & 11 & $(61 \%)$ & \\
\hline (rs161704) & $\mathrm{A} / \mathrm{A}$ & 9 & $(26 \%)$ & 0.87 & 5 & $(29 \%)$ & 4 & $(22 \%)$ & 0.27 \\
\hline IFNG 874T/A & $\mathrm{A} / \mathrm{A}$ & 24 & $(69 \%)$ & & 12 & $(71 \%)$ & 12 & $(67 \%)$ & \\
\hline \multirow[t]{2}{*}{$(\mathrm{rs} 2430561)$} & $\mathrm{A} / \mathrm{T}$ & 9 & $(26 \%)$ & & 5 & $(29 \%)$ & 4 & $(22 \%)$ & \\
\hline & $\mathrm{T} / \mathrm{T}$ & 2 & $(6 \%)$ & 0.38 & 0 & $(0 \%)$ & 2 & $(11 \%)$ & 0.24 \\
\hline IFNGR2 & $\mathrm{A} / \mathrm{A}$ & 10 & $(29 \%)$ & & 3 & $(18 \%)$ & 7 & $(39 \%)$ & \\
\hline Arg64Gln & $\mathrm{A} / \mathrm{G}$ & 18 & $(51 \%)$ & & 9 & $(53 \%)$ & 9 & $(50 \%)$ & \\
\hline (rs9808753) & $\mathrm{G} / \mathrm{G}$ & 7 & $(20 \%)$ & 0.83 & 5 & $(29 \%)$ & 2 & $(11 \%)$ & 0.23 \\
\hline CCL5 $-28 \mathrm{C} / \mathrm{G}$ & $\mathrm{C} / \mathrm{C}$ & 26 & $(74 \%)$ & & 12 & $(71 \%)$ & 14 & $(78 \%)$ & \\
\hline \multirow[t]{2}{*}{ (rs2280788) } & $\mathrm{C} / \mathrm{G}$ & 8 & $(23 \%)$ & & 5 & $(29 \%)$ & 3 & $(17 \%)$ & \\
\hline & $\mathrm{G} / \mathrm{G}$ & 1 & $(3 \%)$ & 0.69 & 0 & $(0 \%)$ & 1 & $(6 \%)$ & 0.37 \\
\hline CCL5 -403G/A & $\mathrm{C} / \mathrm{C}$ & 16 & $(46 \%)$ & & 9 & $(53 \%)$ & 7 & $(39 \%)$ & \\
\hline \multirow[t]{2}{*}{ (rs2107538) } & $\mathrm{C} / \mathrm{T}$ & 14 & $(40 \%)$ & & 7 & $(41 \%)$ & 7 & $(39 \%)$ & \\
\hline & $\mathrm{T} / \mathrm{T}$ & 5 & $(14 \%)$ & 0.51 & 1 & $(6 \%)$ & 4 & $(22 \%)$ & 0.34 \\
\hline CCL11 & $\mathrm{A} / \mathrm{A}$ & 17 & $(49 \%)$ & & 9 & $(53 \%)$ & 8 & $(44 \%)$ & \\
\hline$-384 \mathrm{~A} / \mathrm{G}$ & $\mathrm{A} / \mathrm{G}$ & 15 & $(43 \%)$ & & 6 & $(35 \%)$ & 9 & $(50 \%)$ & \\
\hline (rs17809012) & $\mathrm{G} / \mathrm{G}$ & 3 & $(9 \%)$ & 0.90 & 2 & $(12 \%)$ & 1 & $(6 \%)$ & 0.61 \\
\hline CCL17 & $\mathrm{C} / \mathrm{C}$ & 11 & $(31 \%)$ & & 8 & $(47 \%)$ & 3 & $(17 \%)$ & \\
\hline$-431 \mathrm{C} / \mathrm{T}$ & $\mathrm{C} / \mathrm{T}$ & 14 & $(40 \%)$ & & 5 & $(29 \%)$ & 9 & $(50 \%)$ & \\
\hline$(\mathrm{rs} 223828)$ & $\mathrm{T} / \mathrm{T}$ & 10 & $(29 \%)$ & 0.24 & 4 & $(24 \%)$ & 6 & $(33 \%)$ & 0.14 \\
\hline TSLP -847C/T & $\mathrm{C} / \mathrm{C}$ & 20 & $(57 \%)$ & & 11 & $(65 \%)$ & 9 & $(50 \%)$ & \\
\hline \multirow[t]{2}{*}{ (rs3806933) } & $\mathrm{C} / \mathrm{T}$ & 11 & $(31 \%)$ & & 4 & $(24 \%)$ & 7 & $(39 \%)$ & \\
\hline & $\mathrm{T} / \mathrm{T}$ & 4 & $(11 \%)$ & 0.22 & 2 & $(12 \%)$ & 2 & $(11 \%)$ & 0.61 \\
\hline NGFB & $\mathrm{C} / \mathrm{C}$ & 17 & $(49 \%)$ & & 7 & $(41 \%)$ & 10 & $(56 \%)$ & \\
\hline Ala35Val & $\mathrm{C} / \mathrm{T}$ & 15 & $(43 \%)$ & & 7 & $(41 \%)$ & 8 & $(44 \%)$ & \\
\hline (rs6330) & $\mathrm{T} / \mathrm{T}$ & 3 & $(9 \%)$ & 0.90 & 3 & $(18 \%)$ & 0 & $(0 \%)$ & 0.09 \\
\hline NGFR & $\mathrm{C} / \mathrm{C}$ & 29 & $(83 \%)$ & & 16 & $(94 \%)$ & 13 & $(72 \%)$ & \\
\hline Ser205Leu & $\mathrm{C} / \mathrm{T}$ & 5 & $(14 \%)$ & & 1 & $(6 \%)$ & 4 & $(22 \%)$ & \\
\hline (rs2072446) & $\mathrm{T} / \mathrm{T}$ & 1 & $(3 \%)$ & 0.22 & 0 & $(0 \%)$ & 1 & $(6 \%)$ & 0.17 \\
\hline FCER1A & $\mathrm{T} / \mathrm{T}$ & 30 & $(86 \%)$ & & 16 & $(94 \%)$ & 14 & $(78 \%)$ & \\
\hline$-66 \mathrm{~T} / \mathrm{C}$ & $\mathrm{T} / \mathrm{C}$ & 5 & $(14 \%)$ & & 1 & $(6 \%)$ & 4 & $(22 \%)$ & \\
\hline$(\mathrm{rs} 2251746)$ & $\mathrm{C} / \mathrm{C}$ & 0 & $(0 \%)$ & $\begin{array}{c}0.65 \\
.\end{array}$ & 0 & $(0 \%)$ & 0 & $(0 \%)$ & 0.15 \\
\hline MS4A2 & $\mathrm{T} / \mathrm{T}$ & 19 & $(54 \%)$ & & 11 & $(65 \%)$ & 8 & $(44 \%)$ & \\
\hline$-109 \mathrm{C} / \mathrm{T}$ & $\mathrm{T} / \mathrm{C}$ & 13 & $(37 \%)$ & & 5 & $(29 \%)$ & 8 & $(44 \%)$ & \\
\hline$(\mathrm{rs} 1441586)$ & $\mathrm{C} / \mathrm{C}$ & 3 & $(9 \%)$ & $\begin{array}{r}0.72 \\
\ldots \ldots \ldots \ldots . .\end{array}$ & 1 & $(6 \%)$ & 2 & $(11 \%)$ & $\begin{array}{l}0.48 \\
0.40 .\end{array}$ \\
\hline MS4A2 & $\mathrm{T} / \mathrm{T}$ & 25 & $(71 \%)$ & & 14 & $(82 \%)$ & 11 & $(61 \%)$ & \\
\hline Glu237Gly & $\mathrm{T} / \mathrm{C}$ & 10 & $(29 \%)$ & & 3 & $(18 \%)$ & 7 & $(39 \%)$ & \\
\hline (rs569108) & $\mathrm{C} / \mathrm{C}$ & 0 & $(0 \%)$ & 0.32 & 0 & $(0 \%)$ & 0 & $(0 \%)$ & 0.16 \\
\hline
\end{tabular}


(Table 4) contd.....

\begin{tabular}{|c|c|c|c|c|c|c|c|c|c|}
\hline \multirow{3}{*}{$\begin{array}{l}\text { SNP Name } \\
\text { (SNP ID) } \\
\text { CMA1 }\end{array}$} & \multirow{3}{*}{$\begin{array}{c}\text { Genotype } \\
\text { G/G }\end{array}$} & \multirow{2}{*}{\multicolumn{2}{|c|}{$\frac{\text { Frequency }(\%)}{\text { Total }(\mathbf{n}=\mathbf{3 5})}$}} & \multirow{3}{*}{$\begin{array}{c}P \text { - } \\
\text { Value* }\end{array}$} & \multicolumn{4}{|c|}{ Frequency $(\%)$} & \multirow{3}{*}{$\begin{array}{c}P- \\
\text { Value } \dagger\end{array}$} \\
\hline & & & & & \multicolumn{2}{|c|}{ Responders (n=17) } & \multicolumn{2}{|c|}{ Non-Responders $(n=18)$} & \\
\hline & & 17 & $(49 \%)$ & & 7 & $(41 \%)$ & 10 & $(56 \%)$ & \\
\hline$-1897 \mathrm{G} / \mathrm{A}$ & $\mathrm{G} / \mathrm{A}$ & 16 & $(46 \%)$ & & 9 & $(53 \%)$ & 7 & $(39 \%)$ & \\
\hline (rs1800875) & $\mathrm{A} / \mathrm{A}$ & 2 & $(6 \%)$ & 0.48 & 1 & $(6 \%)$ & 1 & $(6 \%)$ & 0.69 \\
\hline HRH1 -17C/T & $\mathrm{G} / \mathrm{G}$ & 31 & $(89 \%)$ & & 15 & $(88 \%)$ & 16 & $(89 \%)$ & \\
\hline \multirow[t]{2}{*}{ (rs901865) } & $\mathrm{G} / \mathrm{A}$ & 4 & $(11 \%)$ & & 2 & $(12 \%)$ & 2 & $(11 \%)$ & \\
\hline & $\mathrm{A} / \mathrm{A}$ & 0 & $(0 \%)$ & 0.72 & 0 & $(0 \%)$ & 0 & $(0 \%)$ & 0.95 \\
\hline HRH4 & $\mathrm{C} / \mathrm{C}$ & 31 & $(89 \%)$ & & 16 & $(94 \%)$ & 15 & $(83 \%)$ & \\
\hline Ala138Val & $\mathrm{C} / \mathrm{T}$ & 4 & $(11 \%)$ & & 1 & $(6 \%)$ & 3 & $(17 \%)$ & \\
\hline (rs11665084) & $\mathrm{T} / \mathrm{T}$ & 0 & $(0 \%)$ & 0.72 & 0 & $(0 \%)$ & 0 & $(0 \%)$ & 0.31 \\
\hline LTC4S & $\mathrm{A} / \mathrm{A}$ & 21 & $(60 \%)$ & & 11 & $(65 \%)$ & 10 & $(56 \%)$ & \\
\hline$-444 \mathrm{~A} / \mathrm{C}$ & $\mathrm{A} / \mathrm{C}$ & 13 & $(37 \%)$ & & 5 & $(29 \%)$ & 8 & $(44 \%)$ & \\
\hline (rs730012) & $\mathrm{C} / \mathrm{C}$ & 1 & $(3 \%)$ & 0.54 & 1 & $(6 \%)$ & 0 & $(0 \%)$ & 0.35 \\
\hline FLG & $\mathrm{C} / \mathrm{C}$ & 9 & $(26 \%)$ & & 4 & $(24 \%)$ & 5 & $(28 \%)$ & \\
\hline Tyr2194His & $\mathrm{C} / \mathrm{T}$ & 21 & $(60 \%)$ & & 10 & $(59 \%)$ & 11 & $(61 \%)$ & \\
\hline (rs2184953) & $\mathrm{T} / \mathrm{T}$ & 5 & $(14 \%)$ & 0.20 & 3 & $(18 \%)$ & 2 & $(11 \%)$ & 0.85 \\
\hline FLG & $\mathrm{G} / \mathrm{G}$ & 9 & $(26 \%)$ & & 4 & $(24 \%)$ & 5 & $(28 \%)$ & \\
\hline Tyr3105Asp & $\mathrm{G} / \mathrm{T}$ & 26 & $(74 \%)$ & & 13 & $(76 \%)$ & 13 & $(72 \%)$ & \\
\hline (rs2065958) & $\mathrm{T} / \mathrm{T}$ & 0 & $(0 \%)$ & $<0.001$ & 0 & $(0 \%)$ & 0 & $(0 \%)$ & 0.77 \\
\hline FLG & $\mathrm{A} / \mathrm{A}$ & 9 & $(26 \%)$ & & 4 & $(24 \%)$ & 5 & $(28 \%)$ & \\
\hline $13347 \mathrm{G} / \mathrm{A}$ & $\mathrm{A} / \mathrm{G}$ & 21 & $(60 \%)$ & & 10 & $(59 \%)$ & 11 & $(61 \%)$ & \\
\hline (rs12730241) & $\mathrm{G} / \mathrm{G}$ & 5 & $(14 \%)$ & 0.20 & 3 & $(18 \%)$ & 2 & $(11 \%)$ & 0.85 \\
\hline FLG $13586 \mathrm{C} / \mathrm{T}$ & $\mathrm{A} / \mathrm{A}$ & 9 & $(26 \%)$ & & 4 & $(24 \%)$ & 5 & $(28 \%)$ & \\
\hline \multirow[t]{2}{*}{ (rs11204976) } & $\mathrm{A} / \mathrm{G}$ & 21 & $(60 \%)$ & & 10 & $(59 \%)$ & 11 & $(61 \%)$ & \\
\hline & $\mathrm{G} / \mathrm{G}$ & 5 & $(14 \%)$ & 0.20 & 3 & $(18 \%)$ & 2 & $(11 \%)$ & 0.85 \\
\hline
\end{tabular}

$* P$-values were obtained using the Hardy Weinberg equilibrium (HWE) test.

$\uparrow P$-values were obtained using the likelihood ratio chi-square test.

Table 5. Association Between Genotype Combinations and Response to Treatment with Suplatast Tosilate

\begin{tabular}{|c|c|c|c|c|c|}
\hline $\mathrm{T} / \mathrm{T}, \mathrm{T} / \mathrm{C}$ & & & $53.1 \quad(17 / 32)$ & & - \\
\hline \multirow{2}{*}{$\mathrm{C} / \mathrm{C}$} & & $\mathrm{C} / \mathrm{C}, \mathrm{C} / \mathrm{A}$ & $59.3 \quad(16 / 27)$ & & 10.2 \\
\hline & & $\mathrm{A} / \mathrm{A}$ & $12.5 \quad(1 / 8)$ & 0.02 & $(1.1-94.8)$ \\
\hline $\mathrm{C} / \mathrm{C}$ & or & $\mathrm{A} / \mathrm{A}$ & $10.0 \quad(1 / 10)$ & 0.002 & $(1.7-147.5)$ \\
\hline
\end{tabular}

\section{DISCUSSION}

$\mathrm{AD}$ is increasingly recognized as a complex disease, since multifunctional cells and factors interact with each other in its pathogenesis. In the present study, based on the down-regulation of the Th2 inflammatory response by suplatast tosilate, we performed a pilot study to implicate Th2 suppression in the pathogenesis of AD by retrospectively analyzing the polymorphisms of genes reportedly associated with AD or atopic diseases. To this end, patients were recruited from our previous study, in which the efficacy of suplatast tosilate was investigated in adult AD patients whose conditions had been poorly controlled by treatment with topical steroids and adjunct anti-allergic agents, including antihistamines. We then examined the association of suplatast tosilate efficacy and 35 
Table 6. Distribution of IL-4 -590C/T and IL-12B 1188A/C Genotypes Among Japanese and European Populations

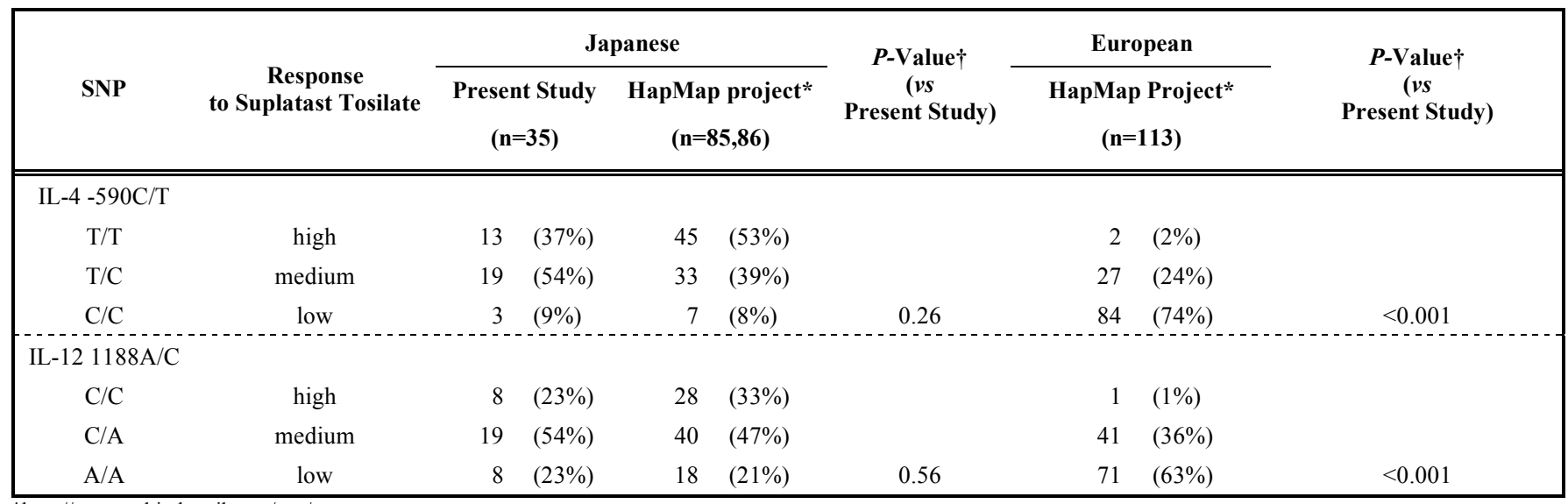

*http://www.ncbi.nlm.nih.gov/snp/

$\dagger P$-values were obtained using the likelihood ratio chi-square test.

SNPs of 27 allergy-related genes. Of note, no significant difference was seen in the minor allele frequencies of each SNP, with the exception of one SNP (Filaggrin Tyr3105Asp) with a low reliability, based on a comparison with an SNP control database for the Japanese population (SNPper or NCBI). These findings suggest that no genetic polymorphisms associated with susceptibility to AD were observed among the SNPs investigated in this study.

The results of the present SNP genotyping analysis clearly indicated that suplatast tosilate efficacy was associated with IL4 and IL-12B polymorphisms, but not with any of the others, indicating that patients with a T/T or T/C genotype for the IL-4 $590 \mathrm{C} / \mathrm{T}$ SNP as well as a C/C or C/A genotype for the IL-12B $1188 \mathrm{~A} / \mathrm{C}$ SNP responded to suplatast tosilate treatment at a significantly higher rate. Moreover, patients with both the IL-4 $590 \mathrm{~T} / \mathrm{T}$ or $\mathrm{T} / \mathrm{C}$ genotype and the IL-12B $1188 \mathrm{C} / \mathrm{C}$ or $\mathrm{C} / \mathrm{A}$ genotype had a significantly higher response rate than those with other genotype combinations. Of note, SNPs in the regulatory sequences of genes are associated with the varying production of relevant cytokines. In fact, the promoter sequence of IL-4 -590T was reported to show a greater binding to nuclear transcription factors than that of $-590 \mathrm{C}[23,24]$. Nakashima et al., reported that PBMC with a $-590 \mathrm{~T} / \mathrm{T}$ or $-590 \mathrm{~T} / \mathrm{C}$ genotype produced higher levels of IL-4 than those with a $-590 \mathrm{C} / \mathrm{C}$ genotype [25]. In general, IL-4 is a major Th2 cytokine that plays an essential role in the class-switching of B cells to IgEproducing cells, Th2 cell differentiation, and the initial phase of tissue inflammation during the Th2-dominant phase of atopic diseases. Recently, IL-4 has been also reported to play an important role in regulating skin homeostasis and innate barrier function in $\mathrm{AD}$ lesions [26]. In fact, Burchard et al., has reported that the sequence variant in the IL-4 promoter region is associated with the asthma FEV1 (Forced expiratory volume in one second) [27]. It is reasonable, therefore, to assume that patients with the IL-4 -590T allele tend to develop a Th2dominated immune response, leading to the susceptibility of these patients to suplatast tosilate which down-regulates Th2related responses including skin manifestations.

Suplatast tosilate has been reported to suppress $\operatorname{IgE}$ formation and eosinophil counts presumably through inhibition of Th2 cytokine in basic experiments and in several clinical studies in patients with atopic asthma [13-20]. However, no significant decreases in Th2-related parameters, such as the total
IgE level and the eosinophil count, were observed after suplatast tosilate treatment even among the responders in this study. The reason for this contradiction is clearly unknown, but, as a possibility, a Th2-dependency may be decreased during the chronic phase of $\mathrm{AD}$ by the effect such as infections. In this sense, of great interest is the observation that patients with the IL-12B $-1188 \mathrm{C} / \mathrm{C}$ genotype responded to suplatast tosilate treatment at a high rate. The IL-12B $-1188 \mathrm{C}$ allele was reported to result in IL-12B mRNA with a lower transcriptional activity and stability than that for the A allele [28]. Although IL-12 p40 encoded by IL-12B is a component of IL-12 (a p40 and p35 heterodimer) [29], the IL-12 p40 homodimer was reported to function as an antagonist of IL-12 action [30, 31]. In fact, PBMC with a $-1188 \mathrm{IL}-12 \mathrm{~B} \mathrm{C} / \mathrm{C}$ genotype having a high response rate to suplatast tosilate reportedly produced significantly higher levels of biologically active IL-12 upon stimulation with LPS or PPD than those of other genotypes with the A allele [32]. IL-12 is known to induce IFN- $\gamma$ production [33], to suppress IgE synthesis [34], and to promote Th1 cell maturation [33]. The results, therefore, suggest that the efficacy of suplatast tosilate is associated with the Th1-immune response that also underlies the AD symptoms. This finding is partly in accordance with an observation by Matsui et al., [35], who reported that PBMC from suplatast tosilate responders with childhood asthma produced higher amounts of IFN- $\gamma$ than those of suplatast tosilate non-responders. The findings are also more directly in accordance with those by Murakami et al., [36], who found that suplatast tosilate treatment significantly suppressed the elevated expression of IL-4, IL-5, and IFN- $\gamma$ mRNA in caspase-1 transgenic mice that spontaneously developed ADlike dermatitis. They also observed that suplatast tosilate treatment significantly decreased the expression of IL-18, which induced Th1 responses in synergy with IL-12, possibly explaining the mechanism of suplatast tosilate efficacy for Th1related responses.

The present results suggest that both Th2- and Th1-related responses may play more important roles in $\mathrm{AD}$ patients who responded to suplatast tosilate treatment than other factors, and ethnic differences may exist in the mechanism of chronic $\mathrm{AD}$, since the allele frequencies of the IL-4 and IL-12B polymorphisms associated with the efficacy of suplatast tosilate differ significantly in the Japanese population compared with the European population. It should be noted, however, that these 
data were based on a retrospective analysis of a small sample size. Therefore, these results have to be confirmed in a largescale prospective study. Moreover, further large-scale investigations taking ethnic differences into account are also needed to clarify the exact mechanism of chronic AD.

\section{CONFLICT OF INTEREST}

This study was funded by Taiho Pharmaceutical Co., Ltd (Tokyo, Japan). Hideki Nagase, Yoshinori Nakachi, Keiji Ishida and Mamoru Kiniwa are employees of Taiho Pharmaceutical Co., Ltd. The other authors declare that they have no other relevant conflicts of interest.

\section{ACKNOWLEDGEMENTS}

We thank Mrs. Saori Inoue and Mr. Yoshihiro Okayama (Taiho Pharmaceutical Co., Ltd., Tokushima, Japan) for technical and statistical assistance, respectively.

\section{REFERENCES}

[1] Cao Y, Liao M, Huang X, Mo Z, Gao F. Meta-analysis of genomewide linkage studies of atopic dermatitis. Dermatitis 2009; 20: 193-9.

[2] Esparza-Gordillo J, Weidinger S, Folster-Holst R, et al. A common variant on chromosome 11q13 is associated with atopic dermatitis. Nat Genet 2009; 41: 596-601.

[3] Holloway JW, Yang IA, Holgate ST. Genetics of allergic disease. J Allergy Clin Immunol 2010; 125: S81-94.

[4] Kawasaki H, Kubo A, Sasaki T, Amagai M. Loss-of-function mutations within the filaggrin gene and atopic dermatitis. Curr Probl Dermatol 2011; 41: 35-46.

[5] Lesiak A, Kuna P, Zakrzewski M, et al. Combined occurrence of filaggrin mutations and IL-10 or IL-13 polymorphisms predisposes to atopic dermatitis. Exp Dermatol 2011; 20: 491-5.

[6] Sun LD, Xiao FL, Li Y, et al. Genome-wide association study identifies two new susceptibility loci for atopic dermatitis in the Chinese Han population. Nat Genet 2011; 43: 690-4.

[7] Katayama I, Kohno Y, Akiyama K, et al. Japanese guideline for atopic dermatitis. Allergol Int 2011; 60: 205-20.

[8] Nishimuta T, Kondo N, Hamasaki Y, Morikawa A, Nishima S. Japanese guideline for childhood asthma. Allergol Int 2011; 60: 147-69.

[9] Ohta K, Yamaguchi M, Akiyama K, et al. Japanese guideline for adult asthma. Allergol Int 2011; 60: 115-45.

[10] Okubo K, Kurono Y, Fujieda S, et al. Japanese guideline for allergic rhinitis. Allergol Int 2011; 60: 171-89.

[11] Miyachi Y, Katayama I, Furue M. Suplatast/tacrolimus combination therapy for refractory facial erythema in adult patients with atopic dermatitis: a meta-analysis study. Allergol Int 2007; 56: 269-75.

[12] Tamaoki J, Kondo M, Sakai N, et al. Effect of suplatast tosilate, a Th2 cytokine inhibitor, on steroid-dependent asthma: a double-blind randomised study. Tokyo Joshi-Idai Asthma Research Group. Lancet 2000; 356: 273-8.

[13] Koda A, Yanagihara Y, Matsuura N. IPD-1151T: a prototype drug for IgE antibody synthesis modulation. Agents Actions Suppl 1991; 34 : 369-78.

[14] Yanagihara Y, Kiniwa M, Ikizawa K, Shida T, Matsuura N, Koda A. Suppression of IgE production by IPD-1151T (suplatast tosilate), a new dimethylsulfonium agent: (2). Regulation of human $\mathrm{IgE}$ response. Jpn J Pharmacol 1993; 61:31-9.

[15] Sano Y, Miyamoto T, Makino S. Anti-inflammatory effect of suplatast tosilate on mild asthma. Chest 1997; 112: 862-3.

[16] Agrawal DK, Cheng G, Kim MJ, Kiniwa M. Interaction of suplatast tosilate (IPD) with chloride channels in human blood eosinophils: a potential mechanism underlying its anti-allergic and anti-asthmatic effects. Clin Exp Allergy 2008; 38: 305-12.
[17] Sano Y, Suzuki N, Yamada H, et al. Effects of suplatast tosilate on allergic eosinophilic airway inflammation in patients with mild asthma. J Allergy Clin Immunol 2003; 111: 958-66.

[18] Myou S, Fujimura M, Kurashima K, et al. Effects of suplatast tosilate, a new type of anti-allergic agent, on airway cough hypersensitivity induced by airway allergy in guinea-pigs. Clin Exp Allergy 2001; 31: 1939-44.

[19] Shiga M, Horiguchi T, Kondo R, et al. Long-term monotherapy with suplatast tosilate in patients with mild atopic asthma: a pilot comparison with low-dose inhaled fluticasone. Asian Pac J Allergy Immunol 2011; 29: 134-42.

[20] Yoshihara S, Ono M, Yamada Y, Fukuda H, Abe T, Arisaka O. Early intervention with suplatast tosilate for prophylaxis of pediatric atopic asthma: a pilot study. Pediatr Allergy Immunol 2009; 20: 486-92.

[21] Kitaba S, Inui S, Matsumoto Y, et al. Examination of an Inhibitory Effect on the Immunoglobulin E Production by Th2 Cytokine Inhibitor on Atopic Dermatitis. Hifu no kagaku 2007; 6 (Suppl 8): 8-14 (in Japanese, with English abstract).

[22] Matsumura Y, Matsumoto Y, Kitaba S, et al. Efficacy of Suplatast Tosilate for Treatment of Atopic Dermatitis Poorly Controlled by Other Anti-allergic Drugs. Hifu no kagaku 2007; 6 (Suppl 8): 1-7 (in Japanese, with English abstract).

[23] Rosenwasser LJ, Klemm DJ, Dresback JK, et al. Promoter polymorphisms in the chromosome 5 gene cluster in asthma and atopy. Clin Exp Allergy 1995; 25 (Suppl 2): 74-8; discussion 95-6.

[24] Song Z, Casolaro V, Chen R, Georas SN, Monos D, Ono SJ. Polymorphic nucleotides within the human IL-4 promoter that mediate overexpression of the gene. J Immunol 1996; 156: 424-9.

[25] Nakashima H, Miyake K, Inoue Y, et al. Association between IL-4 genotype and IL-4 production in the Japanese population. Genes Immun 2002; 3: 107-9.

[26] Sehra S, Yao Y, Howell MD, et al. IL-4 regulates skin homeostasis and the predisposition toward allergic skin inflammation. J Immunol 2010; 184: 3186-90

[27] Burchard EG, Silverman EK, Rosenwasser LJ, et al. Association between a sequence variant in the IL- 4 gene promoter and FEV(1) in asthma. Am J Respir Crit Care Med 1999; 160: 919-22.

[28] Hirota T, Suzuki Y, Hasegawa K, et al. Functional haplotypes of IL$12 \mathrm{~B}$ are associated with childhood atopic asthma. J Allergy Clin Immunol 2005; 116: 789-95.

[29] Kobayashi M, Fitz L, Ryan M, et al. Identification and purification of natural killer cell stimulatory factor (NKSF), a cytokine with multiple biologic effects on human lymphocytes. J Exp Med 1989; 170: 827-45.

[30] Heinzel FP, Hujer AM, Ahmed FN, Rerko RM. In vivo production and function of IL-12 p40 homodimers. J Immunol 1997; 158: 4381-8.

[31] Ling P, Gately MK, Gubler U, et al. Human IL-12 p40 homodimer binds to the IL-12 receptor but does not mediate biologic activity. J Immunol 1995; 154: 116-27.

[32] Yilmaz V, Yentur SP, Saruhan-Direskeneli G. IL-12 and IL-10 polymorphisms and their effects on cytokine production. Cytokine 2005; 30: 188-94

[33] Trinchieri G, Wysocka M, D'Andrea A, et al. Natural killer cell stimulatory factor (NKSF) or interleukin-12 is a key regulator of immune response and inflammation. Prog Growth Factor Res 1992; 4 355-68.

[34] Kiniwa M, Gately M, Gubler U, Chizzonite R, Fargeas C, Delespesse G. Recombinant interleukin-12 suppresses the synthesis of immunoglobulin E by interleukin-4 stimulated human lymphocytes. J Clin Invest 1992; 90: 262-6.

[35] Matsui E, Shinoda S, Fukutomi O, Kaneko H, Fukao T, Kondo N. Relationship between the benefits of suplatast tosilate, a Th2 cytokine inhibitor, and gene polymorphisms in children with bronchial asthma. Exp Ther Med 2010; 1: 977-82.

[36] Murakami T, Yamanaka K, Tokime K, et al. Topical suplatast tosilate (IPD) ameliorates Th2 cytokine-mediated dermatitis in caspasetransgenic mice by downregulating interleukin- 4 and interleukin- 5 . Br J Dermatol 2006; 155: 27-32. 